\title{
Conocimientos, actitudes y prácticas en pacientes con tuberculosis: una revisión sistemática de literatura
}

\section{Knowledge, attitudes and practices in patients with tuberculosis: a systematic literature review}

\author{
Carolina Antolinez-Figueroa ${ }^{1}$ (i) $\otimes$, Eliana-Alejandra Jiménez-Chala ${ }^{1}$ (D) $\otimes$, Paulo-Cesar \\ Caguazango-Atiz ${ }^{1}$ id $\otimes$, Heidy Natalia Urrego-Parra ${ }^{1}$ iD $\otimes$, Lady-Catherine Cantor-Cutiva ${ }^{1}$ \\ (D) $\otimes$, Alba-Idaly Muñoz-Sánchez ${ }^{1}$ (D) $\otimes$ \\ ${ }^{1}$ Grupo de Investigación Saludy Cuidado de los Colectivos; Departamento de Salud de Colectivos; Facultad de Enfermería; Universidad \\ Nacional de Colombia; Bogotá; Colombia.
}

\section{Check for updates}

Correspondencia

Carolina Antolinez-Figueroa.

Email: cantolinezf@unal.edu.co

\section{Citar así}

Antolinez-Figueroa, Carolina; Jiménez-Chala, Eliana-Alejandra; Caguazango-Atiz, PauloCesar; Urrego-Parra, Heidy Natalia; CantorCutiva, Lady-Catherine; Muñoz-Sánchez, Alba-Idaly. (2022). Conocimientos, actitudes y prácticas en pacientes con tuberculosis: una revisión sistemática de literatura. Revista de Investigación e Innovación en Ciencias de la Salud. 4(1), 92-108. https://doi.org/10.46634/riics.77

Recibido: $16 / 07 / 2021$

Revisado: 06/09/2021

Aceptado: 06/11/2021

\section{Editor}

Jorge Mauricio Cuartas Arias, Ph.D. iD

\section{Coeditor}

Fraidy-Alonso Alzate-Pamplona, MSc. (iD

Gopyright $($ 2022. Fundación Universitaria María Cano. La Revista de Investigación e

Innovación en Ciencias de la Salud proporciona acceso abierto a todo su contenido bajo los términos de la licencia Creative Commons Attribution-NonCommercial-NoDerivatives 4.0 International (CC BY-NG-ND 4.0).

\section{Resumen}

Introducción: La tuberculosis es una enfermedad infectocontagiosa de fácil trasmisión. Para su control, los conocimientos, actitudes y prácticas adecuadas son indispensables, así como los factores relevantes para la adherencia al tratamiento.

Objetivo: Caracterizar las definiciones o los conceptos de las dimensiones de conocimientos, actitudes y prácticas en tuberculosis reportados en la literatura durante los últimos diez años.

Materiales y métodos: Se realizó una revisión sistemática de la literatura bajo la metodología "Preferred Reporting Items for Systematic Reviews and Meta-Analyses". Inicialmente, se encontraron 1.720 posibles publicaciones, de las cuales, luego de la aplicación de los criterios de exclusión, quedaron 34 para ser incluidas en el análisis de esta revisión.

Resultados: La mayoría de los estudios fueron publicados en el año 2018 y predominaron las publicaciones en el continente africano. La caracterización de las dimensiones se relaciona así: los conocimientos se definen como características de la enfermedad, las actitudes se relacionan con el estigma social hacia el paciente y las prácticas corresponden con la búsqueda de atención médica por parte del paciente.

Conclusión: Se identificó la escasez de producción científica en donde se especifiquen los conceptos de las dimensiones de conocimientos, actitudes y prácticas, que a su vez permitan el desarrollo de investigaciones de alto rigor y alcance metodológico, ya que las publicaciones realizadas e incluidas para este estudio tuvieron una evaluación de baja calidad. 
Declaración de intereses

Los autores han declarado que no hay conflicto de intereses.

\section{Disponibilidad de datos}

Todos los datos relevantes se encuentran en el artículo. Para mayor información, comunicarse con el autor de correspondencia.

\section{Financiamiento}

Ministerio de Ciencia, Tecnología e Innovación. Convocatoria para el fortalecimiento de proyectos de CTEI en ciencias médicas y de la salud con talento joven e impacto regional. Proyecto de investigación "Estrategia de apropiación social del conocimiento: aplicación móvil para fortalecer la adherencia al tratamiento de la tuberculosis en la ciudad de Bogotá" [Código de proyecto Hermes: 46560]

\section{Descargo de responsabilidad} El contenido de este artículo es responsabilidad exclusiva de los autores y no representa una opinión oficial de sus instituciones ni de la Revista de Investigación e Innovación en Ciencias de la Salud.

\section{Gontribución de los autores} Garolina Antolinez-Figueroa: conceptualización, curación de datos, análisis formal, adquisición de fondos, investigación, metodología, administración de proyecto, recursos, software, supervisión, validación, visualización, escritura: borrador original, escritura: revisión y edición.

Eliana-Alejandra Jiménez-Chala: conceptualización, curación de datos, análisis formal, adquisición de fondos, investigación, metodología, administración de proyecto, recursos, software, supervisión, validación, visualización, escritura: borrador original, escritura: revisión y edición.

Paulo-Cesar Caguazango-Atiz:

conceptualización, curación de datos, análisis formal, adquisición de fondos, investigación, metodología, administración de proyecto, recursos, software, supervisión, validación, visualización, escritura: revisión y edición. Heidy-Natalia Urrego-Parra: conceptualización, curación de datos, análisis formal, adquisición de fondos, investigación, metodología, administración de proyecto, recursos, software, supervisión, validación, visualización, escritura: revisión y edición. Lady-Gatherine Gantor-Gutiva: conceptualización, curación de datos, análisis formal, adquisición de fondos, investigación, metodología, administración de proyecto, recursos, software, supervisión, validación, visualización, escritura: revisión y edición. Alba-Idaly Muñoz-Sánchez: conceptualización, curación de datos, análisis formal, adquisición de fondos, investigación, metodología, administración de proyecto, recursos, software, supervisión, validación, visualización, escritura: revisión y edición.

\section{Palabras clave}

Tuberculosis; infección; pacientes; conocimientos; actitudes; práctica en salud; educación; prevención; control; revisión sistemática.

\section{Abstract}

Introduction: Tuberculosis is an infectious disease of easy transmission. For tuberculosis's control, appropriate knowledge, attitudes, and practices are essential, as well as relevant factors for treatment adherence.

Objective: To characterize the concepts of three dimensions - knowledge, attitudes, and practices - in tuberculosis reported in the literature during the last ten years.

Materials and methods: A systematic review of the literature was carried out under the methodology "Preferred Reporting Items for Systematic Reviews and Meta-Analyzes". Initially, 1.720 possible publications were found, of which, after applying the exclusion criteria, 34 remained to be included in this review.

Results: Most studies were published in 2018, and publications on the African continent predominated. The characterization of the dimensions is related as follows: knowledge was defined as the characteristics of the disease, attitudes were related with the social stigma towards the patient with $\mathrm{TB}$, and practices were associated with seeking medical care by the patients with TB.

Conclusion: There is a lack of publications on the definition of knowledge, attitudes and practices on tuberculosis, which hampers developing high impact research.

\section{Keywords}

Tuberculosis; infection; patients; knowledge; attitudes; health practice; education; prevention; control; systematic review.

\section{Introducción}

En 2019 se reportaron 14.684 casos de tuberculosis (TB) en Colombia, siendo Bogotá una de las ciudades con más casos $(\mathrm{n}=1.218)$ [1]. La tuberculosis es una enfermedad de fácil trasmisión, a través de las gotas de aerosol suspendidas en el aire que trasportan el bacilo de Koch. Estas gotas se trasmiten al toser, estornudar o hablar [2]. Dada la fácil transmisión de la TB, se ha reportado que una persona con tuberculosis activa puede afectar entre 10 a 15 personas [3]. Para el 2020, se estimaron 9.9 millones de casos nuevos de tuberculosis en el mundo, de los cuales el $90 \%$ fueron casos en población adulta económicamente activa [3]. Del total de personas enfermas con tuberculosis, 1.3 millones de personas murieron en el año 2020 a causa de la enfermedad, a lo que deben sumarse otras 251.000 muertes por la coinfección de TB-VIH [3].

A nivel mundial, nacional y local han sido varias las políticas, lineamientos, estrategias y programas diseñados para controlar la TB. Uno de ellos es la "Estrategia fin de la tuberculosis post 2015", en la cual se plantea como objetivo reducir al 95\% la tasa de mortalidad y al 90\% la tasa de incidencia de TB para el año 2035. Adicionalmente, esta estrategia establece como pilar la investigación e innovación intensificada [4]. Algunos estudios han evidenciado que para dar cumplimiento a esta estrategia es 
necesario aumentar los conocimientos, actitudes y prácticas (CAPs) sobre la enfermedad de las personas con tuberculosis, sus familiares y de los trabajadores de la salud [5]. Así mismo, fortalecer los conocimientos sobre la enfermedad mejorará el control, ya que la carencia de conocimientos y de información sobre TB influirá en comportamientos "inadecuados" como la falta de cuidado personal y familiar. Adicionalmente, se disminuirá la estigmatización, el retraso del diagnóstico e inicio del tratamiento, y/o la poca adherencia al mismo [6], aspectos que contribuyen a la continua trasmisión de la enfermedad [7].

Aunque se han realizado diversos estudios sobre los conocimientos, actitudes y prácticas de las personas con tuberculosis pulmonar, no se encontraron en los últimos diez años revisiones sistemáticas de literatura que recopilen y resuman la conceptualización y/o caracterización de estas dimensiones claves para la adherencia al tratamiento. Teniendo en cuenta que las revisiones sistemáticas de literatura permiten organizar información sobre campos de conocimiento ampliamente explorados, realizamos una revisión sistemática de literatura para caracterizar, a partir de la literatura, los CAPs de las personas con tuberculosis en los últimos diez años.

\section{Materiales y métodos}

Se realizó una revisión sistemática de la literatura siguiendo la metodología PRISMA (Preferred Reporting Items for Systematic Reviews and Meta-Analyses) [8]. Se incluyeron seis bases de datos electrónicas: Medline, Pubmed, Science Direct, Web of Science, Scopus y Biblioteca Virtual en Salud. La ecuación de búsqueda utilizada en inglés fue ("Health Knowledge, Attitudes, Practice" AND "Tuberculosis" AND "patient*”); y en español para la base de datos Biblioteca Virtual de la Salud fue ((Conocimientos, Actitudes y Práctica en Salud) AND Tuberculosis AND paciente*). Las ecuaciones de búsqueda se realizaron cruzando únicamente las palabras clave controladas. La búsqueda de la literatura tuvo la siguiente pregunta de investigación: ¿cómo se conceptualizan y/o caracterizan los conocimientos, actitudes y prácticas de personas con tuberculosis en los últimos diez años?

La estrategia de búsqueda fue establecida utilizando los descriptores de ciencias de la salud (DeCS) de la biblioteca virtual en salud (BVS) y Encabezados de Temas Médicos (MeSH: acrónimo de Medical Subject Headings en inglés) de la Biblioteca Nacional de Medicina (NLM en inglés). Se definieron como criterios de inclusión: publicaciones originales en inglés, español y/o portugués; artículos publicados en los últimos diez años (enero 2010 a enero 2020); y artículos que incluyeran los conceptos de por lo menos una dimensión de los CAPs de manera explícita o implícita, es decir, que se especificaran los aspectos evaluados o mencionados dentro de cada dimensión por separado. Para organizar los artículos identificados y excluir duplicados, se utilizó el gestor bibliográfico Zotero® Versión 5.0.89 (Center for History and New Media of the Universidad George Mason).

La selección de los estudios fue realizada por dos investigadores de manera independiente en dos fases (CAF y EAJC). En la primera, los títulos y resúmenes fueron leídos y evaluados a la luz de los criterios de inclusión. Se excluyeron los estudios que no estaban relacionados con la tuberculosis o pacientes con esta enfermedad, así como toda la literatura gris y revisiones de literatura sobre CAPs en comunidades indígenas, trabajadores de la salud y demás temáticas diferentes al objetivo de la revisión.

La segunda fase consistió en la extracción de datos y el análisis bibliométrico de los textos completos, guiados por una matriz elaborada por los investigadores que incluía datos como país, año de publicación, definición de tuberculosis, tipo de diseño del estudio, 
definición de conocimientos, definición de actitudes, definición de prácticas y palabras clave. Los desacuerdos sobre la inclusión de publicaciones en esta fase se resolvieron con un tercer evaluador (LCGG).

Una vez identificados los artículos científicos que cumplían los criterios de inclusión, se procedió a realizar la evaluación de calidad metodológica, para lo cual se utilizó la herramienta "Quality assessment tool for quantitative studies" [9] y su respectivo diccionario [10]. Esta herramienta de evaluación está conformada por ocho ítems principales: sesgo de selección, diseño del estudio, factores de confusión, cegamiento, métodos de recopilación de datos, retiros y abandonos, integridad de la intervención y análisis. Los primeros seis componentes de la herramienta de evaluación cuantitativa se califican mediante una escala del 1 al 3 y la calificación global de cada artículo se obtiene con la misma escala de fuerte, moderado y débil. La evaluación de la calidad metodológica de artículos incluidos se realizó de manera independiente y cegada para evitar interferencias o sesgos. Dos investigadores hicieron esta evaluación; en los casos en que hubo discrepancia, los seis investigadores realizaron la evaluación. Respecto a la evaluación metodológica de los estudios, es importante resaltar que, tras la evaluación de cada artículo por parte de cada uno de los investigadores, se vislumbró que la calidad metodológica del 100\% de los estudios incluidos fue débil.

En total, se identificaron 1.720 estudios potenciales después de excluir los duplicados. Posterior a ello, se revisaron los artículos con base al título y el resumen, identificando 64 documentos para su revisión de texto completo. Finalmente, solo 34 cumplieron con los criterios de inclusión y fueron incluidos para su respectivo análisis (Ver Figura 1).

\section{Resultados}

De los 34 artículos incluidos, el 18\% $(\mathrm{n}=6)$ se publicaron en el año 2018, y otros 6 se publicaron en el 2014 (Ver Figura 2). Frente al continente de publicación, se evidenció que el 53\% de los estudios fueron realizados en el continente africano $(n=18)$ (Ver Figura 3). Así mismo, se identificó que 21 de los estudios eran de tipo cualitativo descriptivo.

Una vez extraídas las características bibliométricas generales de los artículos incluidos, se procedió a establecer categorías temáticas para cada dimensión, las cuales fueron identificadas por dos de los investigadores a partir de la lectura de los estudios.

\section{Conocimientos}

Aunque, solo dos estudios [11,12] reportaron una definición de la dimensión de conocimientos, alrededor del 94\% de los estudios evaluaron e identificaron los temas que constituían esta dimensión. Las definiciones reportadas se sintetizaron de la siguiente manera:

Los conocimientos son una categoría conceptual, y se consideran facilitadores eficaces para contrarrestar las barreras que desencadenan la falta de adherencia al tratamiento, el estigma social y la desconfianza por ingerir los medicamentos para la tuberculosis [11]. Un estudio realizado en Malasia define conocimientos a partir de variables conceptuales sobre terminologías locales de tuberculosis, partiendo del reconocimiento de síntomas, causas, percepciones sobre la atención y efecto de la enfermedad en el paciente y familia [12]. Otro estudio refiere que el desconocimiento sobre la enfermedad crea barreras que impiden que las personas que la padecen entiendan su condición de salud, su enfermedad y así mismo su tratamiento, aspectos fundamentales que pueden poner en peligro esta población [13]. 

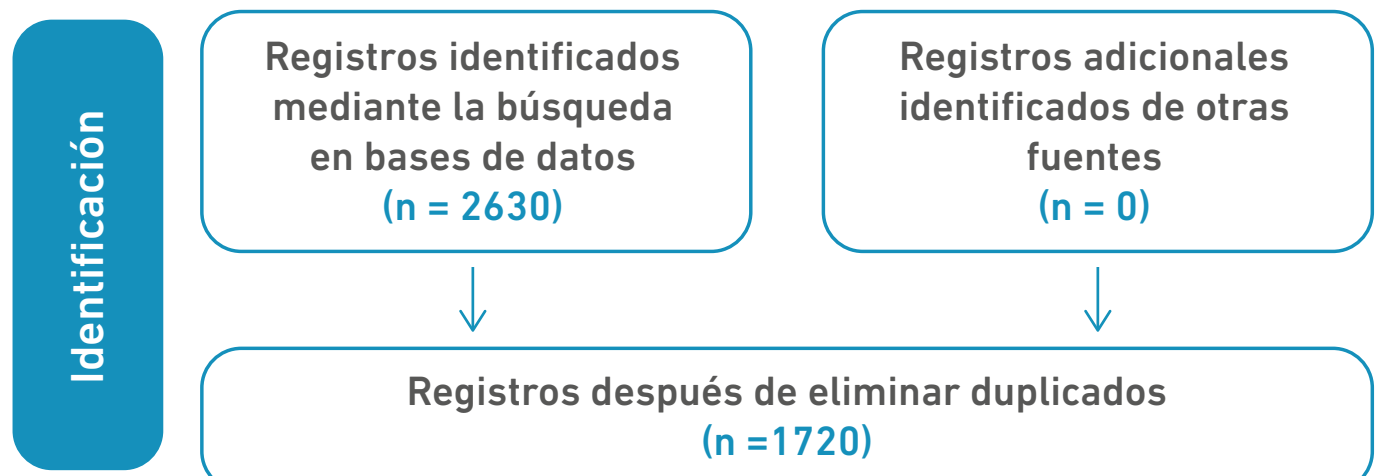

\section{Registros después de eliminar duplicados $(n=1720)$}
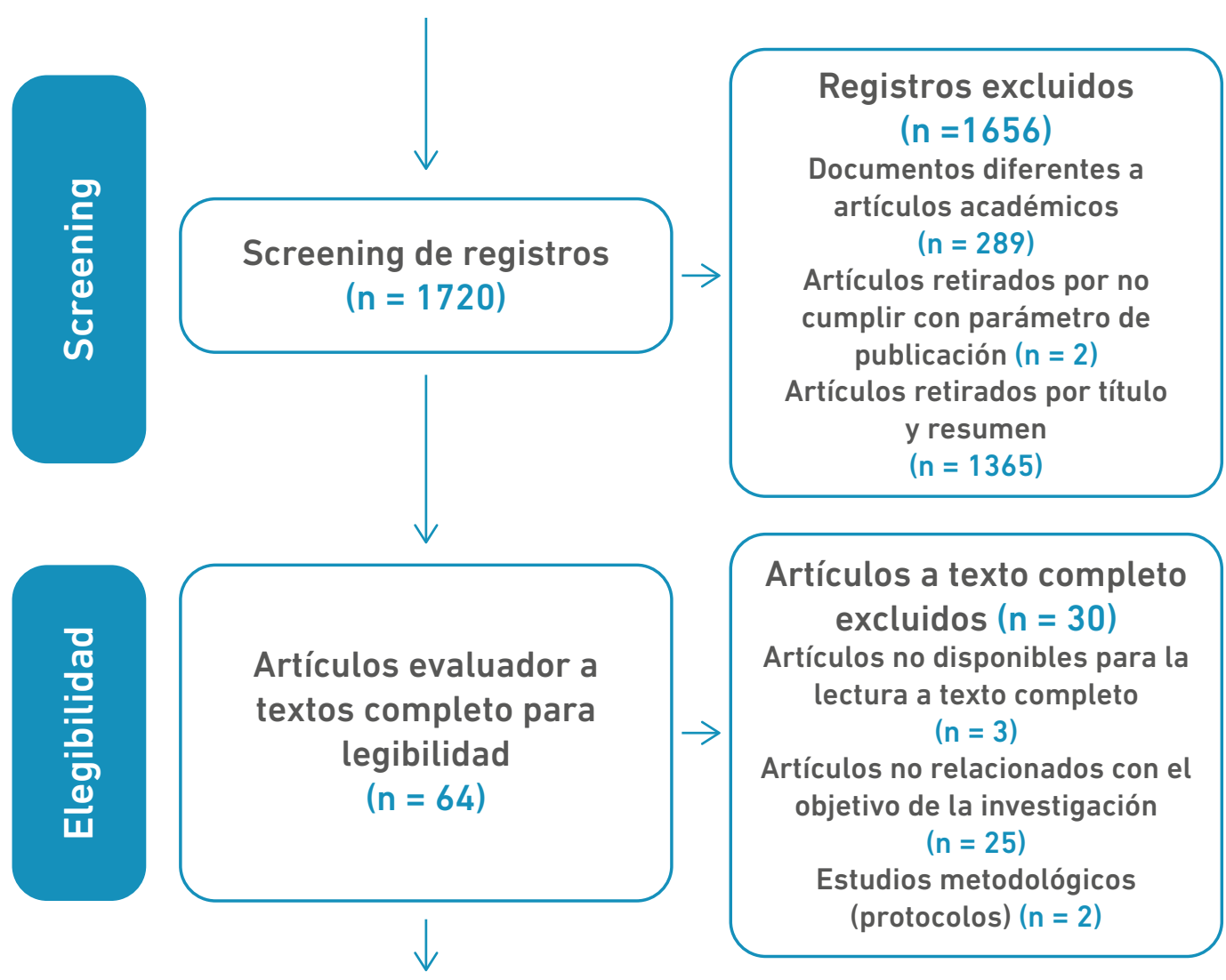

Artículos a texto completo excluidos $(n=30)$

Artículos no disponibles para la lectura a texto completo

$$
(n=3)
$$

Artículos no relacionados con el objetivo de la investigación

$$
\text { ( } n=25)
$$

Estudios metodológicos (protocolos) $(n=2)$

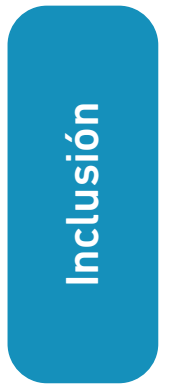

Artículos incluidos en síntesis cualitativa

$$
(n=34)
$$<smiles>C1CCCCC1</smiles>

Artículos incluidos en síntesis cuantitativa (metanálisis)

$$
(n=0)
$$

Figura 1. Diagrama de flujo de metodología sistemática según PRISMA, 2020 


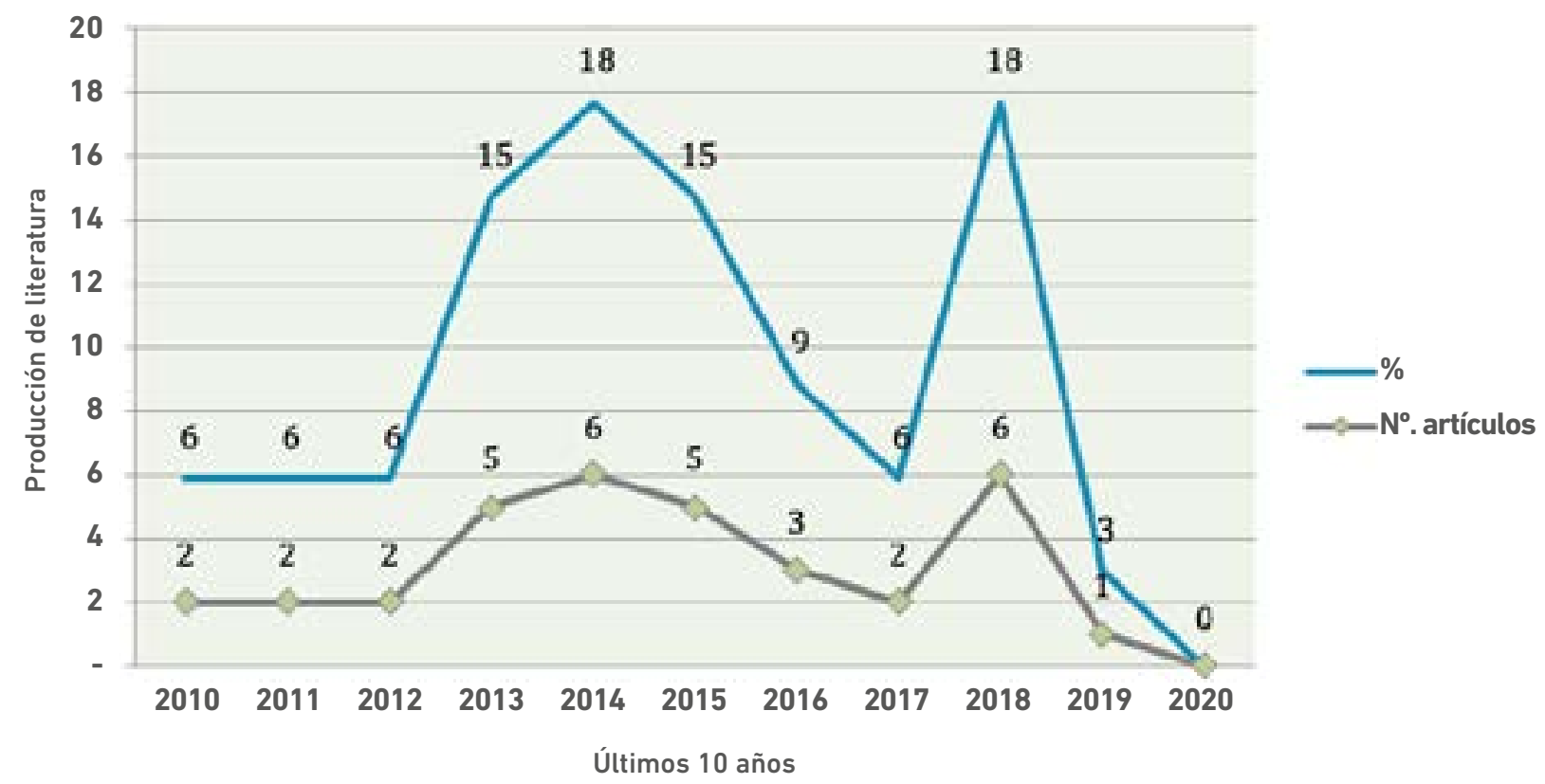

Figura 2. Años por porcentajes de frecuencia de publicación, 2020

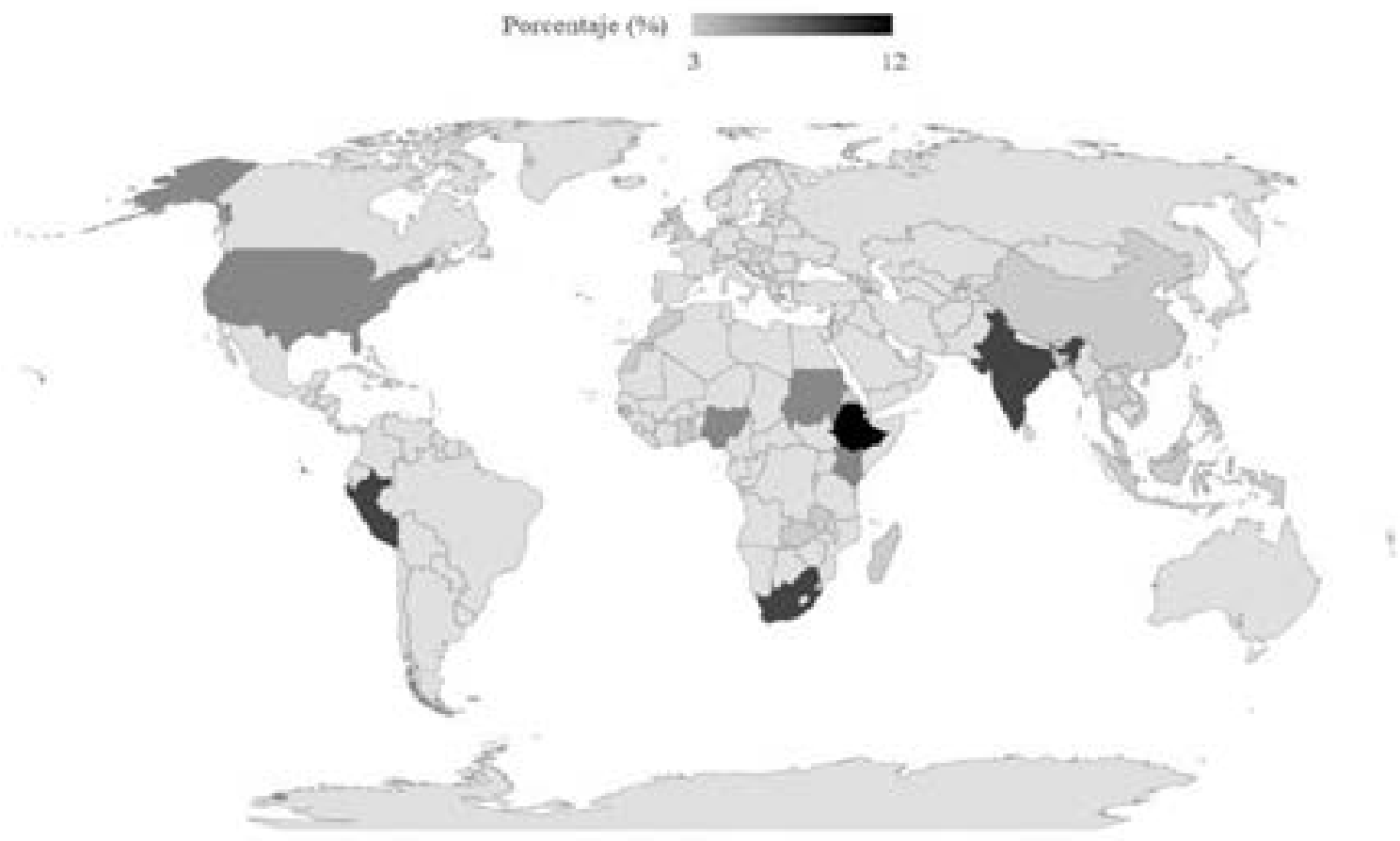

Figura 3. Distribución geográfica de los estudios seleccionados. Los estudios fueron de 21 países de diferentes continentes. Bogotá, Colombia, 2020 
Así mismo, dentro de los estudios que fueron revisados, se evidenció que en el concepto de conocimientos se abordaron las siguientes temáticas: el 71\% de los estudios mencionaron el "modo de transmisión" de la tuberculosis pulmonar, lo que lo hace un aspecto frecuentemente evaluado en esta dimensión. Algunos autores reportaron que los pacientes lo relacionaban con la transmisión por el aire [14], mediante la inhalación de gotas expulsadas a través de la tos y los estornudos [15-18], y el hacinamiento con personas infectadas o animales [18].

En esta misma línea, alrededor del 53\% de los estudios reportaron "signos y síntomas" de la tuberculosis pulmonar como aspectos relevantes para el concepto de conocimientos. El 44\% de los estudios coincidieron en que el síntoma más común de la tuberculosis era la tos persistente y prolongada por dos o más semanas de evolución [15-23]. El esputo con presencia de sangre o hemoptisis también fue asociado como signo de tuberculosis $[15,16,18,20,22]$. Signos como fiebre [16,19-21], fatiga [15,19-21], sudoración nocturna [16,17], pérdida de peso $[15,17,21]$, pérdida de apetito $[17,21]$, dificultad respiratoria $[15,20]$ y el síntoma de debilidad y/o cansancio $[15,16]$ fueron reportados con menor frecuencia.

Cerca del $56 \%$ de los estudios mencionó la "etiología o causas" de la tuberculosis pulmonar como un aspecto de la dimensión de conocimientos. El 37\% de los estudios identificaron que los pacientes reportaron como causa: las bacterias o gérmenes [16-18,22-25], la herencia genética [24,26-30], la suciedad, lo cual incluye alimentos contaminados, agua sin hervir y deficiente higiene corporal [21,31-36].

Los "factores de riesgo" de la tuberculosis pulmonar fueron reportados en el 18\% de los estudios. Algunos de los factores de riesgo reportados fueron: fumar [14,21,22,26,28,35,37], edades extremas (menores de 16 años y mayores de 59 años) [22,35], consumo de sustancias psicoactivas, ser habitante de calle, estar privado de la libertad y ser profesionales de salud [35].

El "tratamiento" de la tuberculosis fue el siguiente tema dentro de la dimensión conocimientos $(\mathrm{n}=24)$. El 21\% de los estudios encontraron que los pacientes refirieron que la tuberculosis pulmonar era curable [14,16,21,29,37]. En uno de estos estudios se afirmó que es curable siempre y cuando se inicie el tratamiento y la atención médica de forma temprana [21]. Con respecto a la duración del tratamiento, el 25\% de los estudios indicaron la importancia de que los pacientes con tuberculosis conocieran cuánto dura el tratamiento $[16,19,21,24,25,28]$. En el 25\% de los estudios se evidenció dentro del tema del tratamiento, los efectos adversos que pueden ocasionar los medicamentos y que pueden interferir en su adherencia $[15,19,20,24,28]$. No obstante, solo el 13\% especificó la duración requerida para completarlo $[21,24,25]$. Finalmente, el 25\% de los artículos reportaron que el tratamiento para la TB era gratuito $[14,18,21,24,29]$.

Alrededor del 29\% de los estudios reportó la "prevención" de la TB como concepto importante de la categoría de conocimientos [14-16,24,28-31,35,38]. Sin embargo, solo cuatro estudios abordaron con especificidad las medidas de prevención que deberían conocer los pacientes con TB, las cuales incluyen el uso del tapabocas durante los primeros quince días del tratamiento, desinfección y ventilación del hogar, higiene de la tos, lavado de manos y uso de pañuelos $[16,24,35,38]$.

El "diagnóstico" para la TB fue mencionado en la dimensión de conocimientos por el 21\% de los estudios $[14,18,19,21,28,35,36,39]$. No obstante, solo un estudio especificó los métodos posibles para el diagnóstico de la tuberculosis pulmonar, entre ellos, el frotis de esputo, el análisis de orina y los rayos $\mathrm{X}[18]$. 
El 15\% de los estudios reportó el tema de "fuente de información". Se resalta la importancia de reconocer cuáles son los medios de comunicación que facilitan obtener suficiente información sobre la enfermedad [21]. Dos estudios coincidieron en que las fuentes de información más reportadas fueron los profesionales de salud; los medios de comunicación audiovisual, como la televisión; y hablar personalmente con personas cercanas, como familiares o amigos $[15,25]$. Un estudio añadió que los medios de comunicación impresos también fueron una fuente valiosa de información para acceder a la información [25]. Los temas anteriormente mencionados fueron descritos en los estudios incluidos y relacionados con el concepto de la dimensión de conocimientos de la TB. Se recalca la importancia de que el paciente debe tener conocimientos adecuados de estos temas para la adherencia de su tratamiento $[13,14,21,26,28]$.

\section{Actitudes}

En términos generales, se entiende la dimensión de actitudes como las percepciones de la enfermedad y su manejo, lo que a su vez determina los comportamientos de búsqueda de atención en salud [27]. Un estudio realizado en el Reino Unido refiere que las actitudes en TB se determinan a partir del estigma, señalamiento y exclusión de los pacientes con esta enfermedad [19]. Otros estudios reportan que las actitudes se explican a partir de las creencias de las personas, y que en la mayoría de las ocasiones desencadenan el rechazo y la desacreditación social de las personas con TB [20,26].

El estigma hacia los pacientes con TB es uno de los temas más frecuentemente abordado. El estigma se define como el proceso por el cual un individuo es devaluado o desacreditado con base a un atributo indeseable [14,33]. También se evidencian atributos de evitación, ocultar diagnóstico, sentimientos y/o emociones de vergüenza, miedo, culpa del paciente con TB [34,40], discriminación y resistencia psicológica [11,13].

En otro estudio realizado en Sudáfrica, los autores describen las actitudes como la percepción de su riesgo, cuidado y tratamiento, opiniones, control de su salud, pensamiento y experiencias sobre el estigma [30] o las percepciones de cambio en el trato de las personas después de haber sido diagnosticados con TB $[12,13,22]$. En los estudios identificados también se hace referencia a que una actitud indiferente o negativa frente al diagnóstico de la TB genera un retraso significativo en el tratamiento de los pacientes [28]. Lo anterior evidencia que las actitudes frente a la enfermedad se ven enmarcadas a partir de las emociones, sentimientos y percepciones de cada uno de los pacientes y que el estigma es uno de los factores que influye de forma negativa en la adherencia al tratamiento contra la TB.

\section{Prácticas}

Ningún autor describió de manera explícita el concepto de la dimensión de prácticas. Sin embargo, el 35\% $(n=12)$ de los estudios identificaron y reportaron aspectos relacionados con esta dimensión.

Alrededor del 21\% de las publicaciones mencionaron la "búsqueda de atención médica" para la TB como un aspecto clave para la construcción de la dimensión de prácticas entre los pacientes con tuberculosis pulmonar. En los artículos incluidos, los pacientes reportaron que el comportamiento más común para la búsqueda de atención médica fue visitar a curanderos tradicionales o espirituales [16,20,22]. Las farmacias locales fueron el segundo lugar más visitado $[16,20]$ y los centros de salud y hospitales fueron el último lugar visitado $[16,18,22]$. 
Las prácticas de "autotratamiento" fueron un aspecto que se reportó en dos estudios. Sin embargo, no se especificaron las acciones que constituyen este aspecto [15,20]. En un estudio realizado en Sudáfrica se afirma que dentro de la dimensión de prácticas se incluyen las acciones que los pacientes están dispuestos a dejar de hacer por estar diagnosticados con TB. Algunos ejemplos fueron: dejar de trabajar durante las dos primeras semanas de tratamiento o hasta que la baciloscopia salga negativa $[38,40]$.

Otros aspectos abordados en esta dimensión fueron la frecuencia de visitar al médico y el retraso en el diagnóstico $[15,41,42]$. Las acciones que permiten la educación frente a la tuberculosis pulmonar y las acciones para la búsqueda de información también fueron aspectos mencionados por un estudio en la dimensión de prácticas $[11,43]$. Las prácticas para prevenir la propagación de la tuberculosis pulmonar [21] y las acciones para el buen control de la enfermedad, como cubrirse la boca y nariz con pañuelos desechables al estornudar, desechar los pañuelos correctamente y lavarse las manos después del contacto con secreciones respiratorias [30] fueron otros aspectos incluidos en la dimensión.

De esta dimensión se evidenció el abordaje de temas como la búsqueda de la atención en salud para el diagnóstico de la enfermedad, la automedicación, la frecuencia de visita al médico para el control de sus síntomas, el retraso en el diagnóstico de la enfermedad, la búsqueda de información sobre TB y las acciones de control de la enfermedad, como lo son la higiene de la tos, desechar la flema en pañuelos y el lavado de manos.

En la Tabla 1 se presenta el resumen de las definiciones de CAPs encontradas en la literatura científica.

\section{Tabla 1. Resumen de las definiciones de CAPs encontradas}

\begin{tabular}{l|c|c|c}
\multicolumn{1}{c|}{ Número de referencia } & \multicolumn{3}{|c}{ Definición abordada } \\
\cline { 2 - 4 } & Conocimientos & Actitudes & Prácticas \\
\hline$[11-28]$ & $X$ & & \\
\hline$[11-14,19,20,22,26,27,28,30,34,40]$ & & $X$ & \\
\hline$[11,15,16,18,20,21,22,30,38,40,41,42,43]$ & & & $X$
\end{tabular}

\section{Discusión}

La presente revisión sistemática tenía como propósito caracterizar las definiciones o conceptos de las dimensiones de conocimientos, actitudes y prácticas de las personas con TB en los últimos diez años. A partir de los estudios revisados, se identificó que los conocimientos son una dimensión que no se define en las investigaciones con frecuencia. Sin embargo, en dos estudios se definió su concepto como una dimensión facilitadora para aumentar la adherencia al tratamiento y disminuir el estigma social, lo cual se relaciona con una mejor calidad de vida de los pacientes y su entorno. Esto va en concordancia con lo que definen los autores de un estudio realizado en Colombia, quienes refieren que los conocimientos en tuberculosis son ideas, información y creencias que tiene una persona, y que pueden afectar las actitudes y predisponer sus actos [44]. 
A pesar de que no se encontró amplia evidencia científica que permita describir el concepto de conocimientos, es importante mencionar que dentro de esta dimensión se encontraron variables de gran importancia para conformar el concepto de los conocimientos en tuberculosis. Estas variables fueron: modo de trasmisión, signos y síntomas, causas, factores de riesgo, tratamiento, prevención, medios diagnósticos y fuente de información de la enfermedad. No obstante, otros autores proponen otros temas de importancia para abordar en esta dimensión, como son el contacto con otras personas dentro del proceso infeccioso de la enfermedad, los efectos adversos de la enfermedad [45] y las comorbilidades con TB [46]. Aunque estos últimos temas son mencionados por algunos autores, no se han utilizado en instrumentos o herramientas sobre conocimientos, pero sí han sido incluidos en apartados sociodemográficos. Es de resaltar que en estos estudios no se identificaron instrumentos validados en su totalidad bajo parámetros psicométricos, lo cual es corroborado por otros autores [47].

Los conocimientos son de vital importancia para determinar las actitudes y las prácticas frente a la enfermedad $[44,46]$. Lo anterior teniendo en cuenta que se ha evidenciado en la literatura científica que, en la mayoría de los casos, los pacientes tienen poco conocimiento sobre su enfermedad y esto a su vez ha ocasionado actitudes negativas y practicas erróneas frente a su enfermedad y durante su tratamiento $[44,45,47]$.

Respecto a la conceptualización de la dimensión de actitudes, no se encontró una definición explícita. Sin embargo, se evidenció que el estigma fue el aspecto más reportado. Este aspecto ha sido reportado por otros autores, quienes concuerdan con que el estigma sobre la TB son percepciones erróneas y negativas de la enfermedad, lo que conlleva a que por miedo no se tomen decisiones oportunas como consultar al centro de salud o a la farmacia más cercana por aparición de alguna sintomatología alusiva a la enfermedad [47], y es por ello que se presentan dificultades para tener un diagnóstico oportuno e iniciar un tratamiento temprano [47]. Así mismo, algunos autores destacan que tener TB representa repercusiones psicológicas, afectando la calidad de vida de las personas con la enfermedad y la finalización exitosa de su tratamiento [48].

Por otro lado, analizar solo el estigma dentro de la dimensión de actitudes deja muy limitada su aproximación, puesto que es de suma importancia abordar otros aspectos actitudinales del individuo y del entorno que confieren cambios a las personas con la enfermedad. Algunos autores coinciden en que, dentro de las dimensiones, es importante mencionar los valores, los sentimientos y las emociones que tienen las personas frente a su enfermedad, aspectos que desde el individuo afectan sus percepciones y formas de actuar para la búsqueda de ayuda y de atención en salud $[44,49]$.

Frente a la dimensión de prácticas, las acciones erróneas para prevenir y controlar la enfermedad pueden ocasionar dificultades en la adherencia al tratamiento, ya que estas acciones se encuentran limitadas por la falta de conocimiento de prácticas que permiten el adecuado comportamiento individual durante el tratamiento, como son las buenas prácticas de higiene de la tos, la asistencia a los centros de salud ante cualquier efecto adverso de los medicamentos, la toma continua y diaria de los medicamentos, etc. Cabe destacar que todos los aspectos reportados son congruentes con la definición de la dimensión de prácticas, la cual corresponde a todas aquellas acciones que realiza el individuo [44], y que provienen del contexto social, familiar e individual, de su aspecto actitudinal y de sus conocimientos [50]. 
A pesar de que en esta revisión no se encontró una definición explícita de cada dimensión, los temas que se abordan dentro de cada uno de ellos permiten tener una aproximación más cercana y confiere la oportunidad de explorarlos a través de las respuestas e información que brinden los sujetos de cada estudio que se realice en la temática de CAPs [43,48,49,51]. Así pues, los CAPs contribuyen al aumento de las probabilidades de éxito de intervenciones para la prevención de la enfermedad [41], puesto que un nivel adecuado de conocimientos influirá en actitudes y prácticas adecuadas para la prevención y control de cualquier enfermedad [5254], así como en la eliminación de barreras de adherencia al tratamiento [55-58].

Teniendo en cuenta lo descrito anteriormente, se podría definir el conocimiento como aquella información o saber que cada individuo posee sobre algún determinado aspecto o situación en salud, y el cual es fundamental para llevar a cabo alguna acción. Las actitudes son aquellas preferencias y predisposiciones que conllevan a la acción de cada persona. Finalmente, las prácticas son aquellos comportamiento o acciones explícitos y observables que cada persona ejecuta para una circunstancia o situación específica.

Con la revisión realizada se logró identificar y caracterizar los temas abordados dentro de las dimensiones de Conocimientos, actitudes y prácticas en tuberculosis, información de gran valor para la elaboración de futuras herramientas o instrumentos de medición de CAPs, que permitan ser replicables y generalizables en diferentes poblaciones, comprendiendo, de este modo, las particularidades de la enfermedad en cada contexto. Por ello es importante continuar con el desarrollo de estudios que aborden la temática, dado que cada estudio será un referente metodológico y conceptual para abordar cada dimensión dentro de las herramientas, encuestas, o instrumentos que se desarrollen para evaluar cada fenómeno de estudio $[59,60]$.

\section{Conclusión}

El presente estudio encontró que para la dimensión de conocimientos se abordaron las características de la enfermedad, del agente infeccioso, del tratamiento, de la prevención de la enfermedad, modos de trasmisión de la enfermedad, entre otros. Para la dimensión de actitudes, se evidenció en la mayoría de los estudios el abordaje del estigma social hacia el paciente con TB; frente a las prácticas, se evidenció el comportamiento de búsqueda de atención médica por parte del paciente con TB.

Los estudios incluidos fueron evaluados mediante la herramienta Quality assessment tool for quantitative studies, identificando el $100 \%$ de los estudios con una calidad metodológica débil-bajo, y siendo en su mayoría estudios cualitativos descriptivos. El presente estudio permitió identificar la necesidad de realizar futuras investigaciones que conceptualicen las dimensiones de CAPs, teniendo en cuenta el contexto sociocultural de cada población. Esta definición de CAPs permitirá a su vez el desarrollo de instrumentos que posibiliten medir los conocimientos, las actitudes y las prácticas de la población con tuberculosis pulmonar.

\section{Referencias}

1. Instituto Nacional de Salud Pública. Boletín Epidemiológico Semanal: Semana epidemiológica 52 [Internet]. [Citado 3 Jul 2020]. Disponible en: https://www.ins.gov.co/buscador-eventos/BoletinEpidemiologico/2019_Boletin_epidemiologico_semana_52.pdf

2. Raviglione MC. Tuberculosis. En: Jameson JL, Fauci AS, Kasper DL, Hauser SL, Longo DL, Loscalzo J, editores. Harrison's Principles of Internal Medicine [Internet]. 20.a Edition. New York, NY: McGraw-Hill Education; 2018 [cited 3 Jul 2020]. Disponible en: accessmedicine.mhmedical.com/content.aspx?aid=1161733805 
3. Organización Mundial de la Salud. Global Tuberculosis Report 2021 [Internet]. [Citado 3 Nov 2021]. Disponible en: file:// / G:/Users/US/Downloads/9789240037021-eng.pdf

4. Organización Mundial de la salud. Estrategia "Fin de la Tuberculosis" post 2015. Últimas actualizaciones 2016 [Internet]. [Citado 3 Jul 2020]. Disponible en: https://www. who.int/tb/Spanish_EndTBStrategy.pdf

5. Mejía J, Quincho-Estares AJ, Riveros M, et al. Conocimientos, actitudes y prácticas sobre tuberculosis en estudiantes de una universidad peruana. Rev Cuba Med Gen Integral [Internet]. 2017 [Citado 3 Jul 2020];33(1):77-89. Disponible en: https://www.researchgate.net/publication/317350820_Conocimientos_actitudes_y_practicas_sobre_tuberculosis_en_estudiantes_de_una_universidad_peruana

6. Zarzuelo R. Conocimiento sobre Tuberculosis en Pacientes que concurren al Hospital SAMCO Dr. Reynaldo Barrionuevo de la cuidad de Capitán Bermudez [Tesis]. Rosario: Universidad Abierta Interamericana; 2012; 47 p..

7. Ladino LE. Creencias y prácticas sobre la tuberculosis en un grupo de pacientes y sus familiares de la ciudad de Bogotá, D. C. Una aproximación cualitativa [Tesis de Maestría] [Internet]. [Bogotá]: Universidad Nacional de Colombia; 2011 [Citado 3 Jul 2020].

8. Moher D, Liberati A, Tetzlaff J, Altman DG, Group TP. Preferred Reporting Items for Systematic Reviews and Meta-Analyses: The PRISMA Statement. PLOS Med [Internet]. 2009 [cited 3 Jul 2020];6(7):e1000097. Disponible en: https://journals.plos.org/ plosmedicine /article?id=10.1371/journal.pmed.1000097

9. Thomas H, Ciliska D, Dobbins M, Micucci S. Quality assessment tool for quantitative studies. Effective Public Health Practice Project. Toronto: McMaster University; 2003.

10. Thomas H, Ciliska D, Dobbins M, Micucci S. Quality assessment tool for quantitative studies dictionary: the Effective Public Health Practice Project (EPHPP). [Toronto]: McMaster University [Internet]; 2008. [cited 3 Jul 2020]. Disponible en: https://www. ephpp.ca/PDF/QADictionary_dec2009.pdf

11. McNally TW, De Wildt G, Meza G, Wiskin GMD. Improving outcomes for multi-drug-resistant tuberculosis in the Peruvian Amazon - A qualitative study exploring the experiences and perceptions of patients and healthcare professionals. BMC Health Serv Res [Internet]. 2019 [cited 3 Jul 2020];19(1). Disponible en: https://www.scopus.com/inward/ record.uri?eid=2-s2.0-8507 1331959\&doi=10.1186\%2fs 12913-019-4429-y\&partnerI$\mathrm{D}=40 \& \mathrm{md} 5=17834 \mathrm{~b} 18 \mathrm{a} 0 \mathrm{bc5f1d93a313b999ad3b88}$

12. Rundi C. Understanding Tuberculosis: Perspectives and Experiences of the People of Sabah, East Malaysia. J Health Popul Nutr [Internet]. 2010 [cited 3 Jul 2020];28(2):114-23. Disponible en: https://pubmed.ncbi.nlm.nih.gov/20411673/

13. Dodor EA. The feelings and experiences of patients with tuberculosis in the Sekondi-Takoradi Metropolitan district: implications for TB control efforts. Ghana Med J [Internet]. 2012 [cited 3 Jul 2020];46(4):211-8. Disponible en: https://pubmed.ncbi.nlm. nih.gov/23661839/ 
14. Suleiman MMA, Sahal N, Sodemann M, El Sony A, Aro AR. Tuberculosis stigma in Gezira State, Sudan: a case-control study. Int J Tuberc Lung Dis [Internet]. 2013 [cited 3 Jul 2020];17(3):388-93. Disponible en: https://pubmed.ncbi.nlm.nih.gov/23407228/

15. Esmael A, Ali I, Agonafir M, Desale A, Yaregal Z, Desta K. Assessment of patients' knowledge, attitude, and practice regarding pulmonary tuberculosis in Eastern Amhara Regional State, Ethiopia: Cross-sectional study. Am J Trop Med Hyg [Internet]. 2013 [cited 3 Jul 2020];88(4):785-8. Disponible en: https://pubmed.ncbi.nlm.nih.gov/23419364/

16. Badane AA, Dedefo MG, Genamo ES, Bekele NA. Knowledge and Healthcare Seeking Behavior of Tuberculosis Patients attending Gimbi General Hospital, West Ethiopia. Ethiop J Health Sci [Internet]. 2018 [cited 3 Jul 2020];28(5):529-38 Disponible en: https://www.ncbi.nlm.nih.gov/pmc/articles/PMC6308772/

17. Seyoum A, Legesse M. Knowledge of tuberculosis (TB) and human immunodeficiency virus (HIV) and perception about provider initiated HIV testing and counselling among TB patients attending health facilities in Harar town, Eastern Ethiopia. BMC Public Health [Internet]. 2013 [cited 3 Jul 2020];13(1). Disponible en: https://www.ncbi.nlm. nih.gov/pmc/articles/PMC6308772/

18. Zhang Z, Xia D, Zhang X, Li X, Ma J, Ding S, et al. Concerns about the knowledge, attitude and practice of tuberculosis in Anqing, China: comparison between new tuberculosis patients and non-tuberculosis patients. Biomed Res-India [Internet]. 2016 [cited 3 Jul 2020];27(4):1337-47. Disponible en: https://www.alliedacademies.org/articles/concerns-about-the-knowledge-attitude-and-practice-of-tuberculosis-in-anqing-china-comparison-between-new-tuberculosis-patients-an.html

19. Craig GM, Joly LM, Zumla A. 'Complex’ but coping: experience of symptoms of tuberculosis and health care seeking behaviours - a qualitative interview study of urban risk groups, London, UK. BMC Public Health [Internet]. 2014 [cited 3 Jul 2020];14(618):1-9. Disponible en: https://bmcpublichealth.biomedcentral.com/track/pdf/10.1186/14712458-14-618.pdf

20. Ayisi JG, Van'T Hoog AH, Agaya JA, McHembere W, Nyamthimba PO, Muhenje O, et al. Care seeking and attitudes towards treatment compliance by newly enrolled tuberculosis patients in the district treatment programme in rural western Kenya: A qualitative study. BMC Public Health [Internet]. 2011 [cited 3 Jul 2020];11(515):1-10. Disponible en: https://bmcpublichealth.biomedcentral.com/track/pdf/10.1186/1471-2458-11-515.pdf

21. Rakotosamimanana S, Mandrosovololona V, Rakotonirina J, Ramamonjisoa J, Ranjalahy JR, Randremanana RV, et al. Spatial analysis of pulmonary tuberculosis in antananarivo madagascar: Tuberculosis-related knowledge, attitude and practice. Plos One [Internet]. 2014 [cited 3 Jul 2020];9(11). Disponible en: https://bmcpublichealth.biomedcentral. com/track/pdf/10.1186/1471-2458-11-515.pdf

22. Viney KA, Johnson P, Tagaro M, Fanai S, Linh NN, Kelly P, et al. Tuberculosis patients' knowledge and beliefs about tuberculosis: A mixed methods study from the Pacific Island nation of Vanuatu. BMC Public Health [Internet]. 2014 [cited 3 Jul 2020];14(1). Disponible en: https://www.scopus.com/inward/record.uri? eid=2-s2.0-84901639347 $\&$ doi $=10.1186 \% 2 f 147$ 1-2458-14-467\&partnerID=40\&md5=5d05a22cbe311 e645e8e$85411 \mathrm{~b} 11493 \mathrm{~b}$ 
23. Tachfouti N, Slama K, Berraho M, Nejjari G. The impact of knowledge and attitudes on adherence to tuberculosis treatment: a case-control study in a Moroccan region. Pan Afr Med J [Internet]. 2012 [cited 3 Jul 2020];12(1):52-52. Disponible en: https://www.ncbi. nlm.nih.gov/pmc/articles/PMC3428172/

24. Pai M, Delavallade C, Huddart S, Bossuroy T, Pons V, Baral S. Knowledge about tuberculosis and infection prevention behavior: A nine city longitudinal study from India. Plos One [Internet]. 2018 [cited 3 Jul 2020];13(10). Disponible en: https://www. scopus.com/inward/record.uri?eid=2-s2.0-85055640755\&doi=10.1371\%2fjournal.pone.0206245\&partnerID $=40 \& \operatorname{md} 5=2734 \mathrm{dd} 12 \mathrm{a} 04740 \mathrm{c} 22 \mathrm{~b} 72095 \mathrm{aae} 2 \mathrm{a} 8 \mathrm{c} 19$

25. MaharajJ, Ross A, Maharaj NR, Campbell L. Multidrug-resistant tuberculosis in KwaZulu-Natal, South Africa: An overview of patients' reported knowledge and attitudes. Afr J Prim Health Gare Fam Med [Internet]. 2016 [cited 3 Jul 2020];8(1):1-6. Disponible en: https://www.semanticscholar.org/paper/Multidrug-resistant-tuberculosis-in-KwaZulu-Natal\%2C-Maharaj-Ross/090dab8b377ce2f24d48ad9482e74f555831658e

26. Cremers AL, De Laat MM, Kapata N, Gerrets R, Klipstein-Grobusch K, Grobusch MP. Assessing the consequences of stigma for tuberculosis patients in urban Zambia. PLoS ONE [Iternet]. 2015 [cited 3 Jul 2020];10(3). Disponible en: https://www.scopus.com/inward/record.uri? eid=2-s2.0-84926203727\&doi=10.1371\%2fjournal.pone.0119861\&partnerID $=40 \& \mathrm{md} 5=\mathrm{c} 7 \mathrm{~b} 78 \mathrm{f} 4500 \mathrm{fc} 14 \mathrm{~d} 1 \mathrm{ea} 9150481 \mathrm{f063b9c}$

27. Isara AR, Akpodiete A. Concerns about the knowledge and attitude of multidrug-resistant tuberculosis among health care workers and patients in Delta State, Nigeria. Niger J Clin Pract [Internet]. 2015 [cited 3 Jul 2020];18(5):664-9. Disponible en: https://pubmed.ncbi.nlm.nih.gov/26096247/

28. Mbuthia GW, Olungah CO, Ondicho TG. Knowledge and perceptions of tuberculosis among patients in a pastoralist community in Kenya: A qualitative study. Pan Afr MedJ [Internet]. 2018 [cited 3 Jul 2020];30(1). Disponible en: https://www.scopus.com/inward/ record. uri? eid $=2$-s2.0-85055487802\&doi $=10.11604 \% 2$ fpamj.2018.30.287.14836\&partnerID $=40 \&$ md5 $=72 a 920$ daa918796a89b9e803fe4289ef

29. Mondal MN, Nazrul HM, Chowdhury MRK, Howard J. Socio-demographic factors affecting knowledge level of tuberculosis patients in Rajshahi city, Bangladesh. Afr Health Sci [nternet]. 2014 [cited 3 Jul 2020];14(4):855-65. Disponible en: https://pubmed. ncbi.nlm.nih.gov/25834494/

30. Kigozi NG, Heunis JC, Engelbrecht MC, van Rensburg APJ, van Rensburg HCJD. Tuberculosis knowledge, attitudes and practices of patients at primary health care facilities in a South African metropolitan: research towards improved health education. BMC Public Health [Internet]. 2017 [cited 3 Jul 2020];17(795). Disponible en: https://www.ncbi. nlm.nih.gov/pmc/articles/PMC5633895/

31. Laurante J, Remuzgo Artezano F, Gallardo Cartagena JA, Taype Medina L, Huapaya Torres J, Carrillo Bermudez JF, et al. Conocimiento y actitudes acerca de la transmisión y prevención de la tuberculosis en pacientes con tuberculosis multidrogorresistente. Rev Peru Epidemiol [Internet]. 2010 [Citado 3 Jul 2020];14(1):1-7. Disponible en: https:// www.redalyc.org/pdf/2031/203119805005.pdf 
32. Colson PW, Gouzens GL, Royce RA, Kline T, Chavez-Lindell T, Welbel S, et al. Examining the Impact of Patient Characteristics and Symptomatology on Knowledge, Attitudes, and Beliefs Among Foreign-born Tuberculosis Cases in the US and Canada. J Immigr Minor Health [Internet]. 2014 [cited 3 Jul 2020];16(1):125-35. Disponible en: https://pubmed.ncbi.nlm.nih.gov/23440450/

33. Mukerji R, Turan JM. Exploring manifestations of tb-related stigma experienced by women in Kolkata, India. Ann Glob Health [Internet]. 2018 [cited 3 Jul 2020];84(4):727-35. Disponible en: https://annalsofglobalhealth.org/articles/10.29024/aogh.2383/

34. Dewi C, Barclay L, Passey M, Wilson S. Improving knowledge and behaviours related to the cause, transmission and prevention of Tuberculosis and early case detection: A descriptive study of community led Tuberculosis program in Flores, Indonesia. BMC Public Health [Internet]. 2016 [cited 3 Jul 2020];16(1). Disponible en: https:// www.scopus.com/inward/record.uri? eid $=2$-s2.0-84982943737\&doi $=10.1186 \%$ 2fs 12889-016-3448-4\&partnerID=40\&md5=bd6e8ac131d3a0d285f66532625ab2aa

35. Royce RA, Colson PW, Woodsong C, Swinson-Evans T, Walton W, Maiuri A, et al. Tuberculosis knowledge, awareness, and stigma among African-Americans in three Southeastern Counties in the USA: A qualitative study of community perspectives. J Racial Ethn Health Disparities [Internet]. 2015 [cited 3 Jul 2020];4(1):47-58. Disponible en: https://www.ncbi.nlm.nih.gov/pmc/articles/PMC4927396/

36. Gebreweld FH, Kifle MM, Gebremicheal FE, Simel LL, Gezae MM, Ghebreyesus SS, et al. Factors influencing adherence to tuberculosis treatment in Asmara, Eritrea: a qualitative study. J Health Popul Nutr [Internet]. 2018 [cited 3 Jul 2020];37(1). Disponible en: https://pubmed.ncbi.nlm.nih.gov/29304840/

37. Westerlund EE, Tovar MA, Lonnermark E, Montoya R, Evans CA. Tuberculosis-related knowledge is associated with patient outcomes in shantytown residents; results from a cohort study, Peru. J Infect [Internet]. 2015 [cited 3 Jul 2020];7 1(3):347-57. Disponible en: https://pubmed.ncbi.nlm.nih.gov/26033695/

38. Gonzalez-Angulo Y, Geldenhuys H, Van As D, Buckerfield N, Shea J, Mahomed H, et al. Knowledge and acceptability of patient-specific infection control measures for pulmonary tuberculosis. Am J Infect Control [Internet]. 2013 [cited 3 Jul 2020];41(8):717-22. Disponible en: https://pubmed.ncbi.nlm.nih.gov/23375576/

39. DeLuca A, Dhumal G, Paradkar M, Suryavanshi N, Mave V, Kohli R, et al. Addressing knowledge gaps and prevention for tuberculosis-infected Indian adults: A vital part of elimination. BMC Infect Dis [Internet]. 2018 [cited 3 Jul 2020];18(1). Disponible en: https://www.scopus.com/inward/record.uri?eid=2-s2.0-85046496843\&doi=10.1186\%2fs 12879-018-3116-7\&partnerID=40\&md5=b4e848daf586a175a4bc171f4a951000

40. Kipp AM, Pungrassami P, Nilmanat K, Sengupta S, Poole C, Strauss RP, et al. Socio-demographic and AIDS-related factors associated with tuberculosis stigma in southern Thailand: A quantitative, cross-sectional study of stigma among patients with TB and healthy community members. BMC Public Health [Internet]. 2011 [cited 3 Jul 2020];11(1). Disponible en: https://www.scopus.com/inward/record.uri? eid=2-s2.0-80 052120095\&doi $=10.1186 \% 2$ f147 1-2458-11-675\&partnerID=40\&md5=1e23bfdaebc$79128 \mathrm{df} 50680 \mathrm{e} 0 \mathrm{c} 5 \mathrm{~d} 0139$ 
41. Fox GJ, Loan LP, Nhung NV, Loi NT, Sy DN, Britton WJ, et al. Barriers to adherence with tuberculosis contact investigation in six provinces of Vietnam: A nested case-control study. BMC Infect Dis [Internet]. 2015 [cited 3 Jul 2020];15(1). Disponible en: https://www.scopus.com/inward/record.uri?eid=2-s2.0-84926382477\&doi=10.1186\%2fs 12879-015-0816-0\&partnerID=40\&md5=a367b7ec5cd48407f84014357b2a01af

42. Biya O, Gidado S, Abraham A, Waziri N, Nguku P, Nsubuga P, et al. Knowledge, care-seeking behavior, and factors associated with patient delay among newly-diagnosed pulmonary tuberculosis patients, Federal Capital Territory, Nigeria, 2010. Pan Afr Med J [Internet]. 2014 [cited 3 Jul 2020];18(6). Disponible en: https://www.ncbi.nlm.nih.gov/ pmc/articles/PMC4199348/

43. Tola HH, Garmaroudi G, Shojaeizadeh D, Tol A, Yekaninejad MS, Ejeta LT, et al. The Effect of Psychosocial Factors and Patients' Perception of Tuberculosis Treatment Non-Adherence in Addis Ababa, Ethiopia. Ethiop J Health Sci [Internet]. 2017 [cited 3 Jul 2020];27(5):447-58. Disponible en: https://pubmed.ncbi.nlm.nih.gov/29217949/

44. Muñoz-Sánchez AI, Rubiano-Mesa YL, Saavedra-Cantor CJ. Measuring instrument: Knowledge, attitudes and practices of people with pulmonary tuberculosis. Rev Lat Am Enfermagem [Internet]. 2019 [cited 3 Jul 2020];27(1). Disponible en: https://www.scopus.com/inward/record.uri? eid=2-s2.0-85060790820\&doi $=10.1590 \% 2 \mathrm{f15} 18-8345.260$ 8.3086\&partnerID=40\&md5=1 ab80db97b323cfabba26b934c01 ebe7

45. Higuita-Gutiérrez LF, Arango-Franco CA, Cardona-Arias JA, Higuita-Gutiérrez LF, Arango-Franco CA, Cardona-Arias JA. Factores de riesgo para la infección por tuberculosis resistente: Metanálisis de estudios de casos y controles. Rev Esp Salud Pública [Internet]. 2018 [citado 16 Jul 2020];92(1). Disponible en: http://scielo.isciii.es/scielo. php?script=sci_abstract\&pid=S1 135-57272018000100505\&lng=es\&nrm=iso\&tlng=en

46. Abarca Tomás B, Pell C, Bueno Cavanillas A, Guillén Solvas J, Pool R, Roura M. Tuberculosis in migrant populations. A systematic review of the qualitative literature. PloS One [Internet]. 2013 [cited 16 Jul 2020];8(12):e82440. Disponible en: https://pubmed.ncbi. nlm.nih.gov/24349284/

47. Duarte MD, Morais RB de, Marziale MHP. Instrumentos e fatores impactantes sobre o conhecimento das medidas de precauções-padrão entre trabalhadores de saúde. Enferm Glob [Internet]. 2016 [cited 16 Jul 2020];15(1):272-321. Disponible en: http://scielo. isciii.es/pdf/eg/v15n41/pt_revision1.pdf

48. Giraldo Gallego N, Valencia Acevedo D, Cardona-Arias JA. Calidad de vida relacionada con la salud en tuberculosis: Revisión sistemática y metanálisis. Infectio [Internet]. 2018 [citado 16 Jul 2020];22(3):124. Disponible en: http://www.scielo.org:co/pdf/inf/ v22n3/0123-9392-inf-22-03-00124.pdf

49. Antolinez Figueroa Carolina C, Bello Velásquez MC, Romero Bernal LF, Muñoz Sánchez AI. Instrumentos y herramientas de evaluación sobre conocimientos de tuberculosis. Enferm Glob [Internet]. 2017 [citado 16 Jul 2020];16(48):499-514. Disponible en: http://scielo.isciii.es/pdf/eg/v16n48/1695-6141-eg-16-48-00499.pdf

50. Ariztía T. La teoría de las prácticas sociales: particularidades, posibilidades y límites. Cinta Moebio [Internet]. 2017 [citado 16 Jul 2020];1(59). Disponible en: https://www.moebio.uchile.cl/59/ariztia.html 
51. Nasiri A, Balouchi A, Rezaie-Keikhaie K, Bouya S, Sheyback Mahmood, Rawajfah OA. Knowledge, attitude, practice, and clinical recommendation toward infection control and prevention standards among nurses: A systematic review. Am J Infect Control [Internet]. 2019 [cited 16 Jul 2020];47(7):827-33. Disponible en: https://pubmed.ncbi.nlm.nih. gov/30612817/

52. Quaye B, Alatrash M, Metoyer CE. Changes in knowledge, attitudes and beliefs in BSN students after SBIRT education and practice in home health.J Prof Nurs [Internet]. 2020 [cited 8 Dec 2020];36(6):649-58. Disponible en: http://www.sciencedirect.com/science/ article/pii/S8755722320301745

53. Siegel SD, Laurenceau JP, Hill N, Bauer A-M, Flitter A, Ziedonis D, et al. Assessing barriers to providing tobacco use disorder treatment in community mental health settings with a revised version of the Smoking Knowledge, Attitudes, and Practices (S-KAP) instrument. Addict Behav [Internet]. 2020 [cited 8 Dec 2020];1(114):106735.

54. Lipsey AF, Waterman AD, Wood EH, Balliet W. Evaluation of first-person storytelling on changing health-related attitudes, knowledge, behaviors, and outcomes: A scoping review. Patient Educ Couns [Internet]. 2020 [cited 8 Dec 2020];103(10):1922-34. Disponible en: https://pubmed.ncbi.nlm.nih.gov/32359877/

55. Wang Y, Guo F, Wei J, Zhang Y, Liu Z, Huang Y. Knowledge, attitudes and practices in relation to antimicrobial resistance amongst Chinese public health undergraduates. J Glob Antimicrob Resist [Internet]. 2020 [cited 8 Dec 2020];23(1):9-15. Disponible en: https://www.sciencedirect.com/science/article/pii/S2213716520301995

56. Eskandari F, Abdullah KL, Zainal NZ, Wong LP. The effect of educational intervention on nurses' knowledge, attitude, intention, practice and incidence rate of physical restraint use. Nurse Educ Pract [Internet]. 2018 [cited 8 Dec 2020];32(1):52-7. Disponible en: https://pubmed.ncbi.nlm.nih.gov/30029085/

57. Tay C, Yuh AS, Sheau Lan EL, Ong CE, Aloweni F, Lopez V. Development and validation of the incontinence associated dermatitis knowledge, attitude and practice questionnaire. J Tissue Viability [Internet]. 2020 [cited 8 Dec 2020];29(4):244-51. Disponible en: https://www.sciencedirect.com/science/article/abs/pii/S0965206X20300826

58. Ventura-León JL, Arancibia M, Madrid E, Ventura-León JL, Arancibia M, Madrid E. La importancia de reportar la validez y confiabilidad en los instrumentos de medición: Comentarios a Arancibia et al. Rev Médica Chile [Internet]. 2017 [citado 8 Dic 2020];145(7):955-6. Disponible en: https://scielo.conicyt.cl/scielo.php?script=sci_arttext\&pid=S0034-98872017000700955

59. Qasim M, Awan UA, Afzal MS, Saqib MAN, Siddiqui S, Ahmed H. Dataset of knowledge, attitude, practices and psychological implications of healthcare workers in Pakistan during COVID-19 pandemic. Data Brief [Internet]. 2020 [cited 8 Dec 2020];32(1):106234. Disponible en: https://pubmed.ncbi.nlm.nih.gov/32895632/

60. Vasconcelos GTM, Silva Gomes ML, Ribeiro GL, Oriá MOB, Geoffrion R, Vasconcelos Neto JA. Women and healthcare providers' knowledge, attitudes and practice related to pessaries for pelvic organ prolapse: A Systematic review. Eur J Obstet Gynecol Reprod Biol [Internet]. 2020 [cited 8 Dec 2020];247(1):132-42. Disponible en: https://pubmed. ncbi.nlm.nih.gov/32113060/ 